\title{
Accessing the Influence of Hess-Murray Law on Suspension Flow through Ramified Structures
}

\author{
Ana Serrenho, $0^{1,2, a}$ and Antonio F. Miguel ${ }^{2, b^{*}}$ \\ ${ }^{1}$ Earth Institute, University College Dublin, Belfield, Dublin 4, Ireland \\ ${ }^{2}$ Geophysics Center CGE and Department of Physics, University of Évora, Portugal

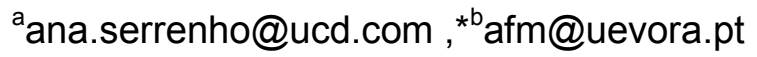

Keywords: ramified structure, Hess-Murray law, constructal law, fluid flow, suspension flow.

\begin{abstract}
The present study focuses on fluid flow and particle transport in symmetric T-shaped structures formed by tubes with circular and square cross-section. The performances of optimized structures (i.e., structures designed based on constructal allometric laws for minimum flow resistance) and not optimized structures were studied. Flow resistance and particle penetration efficiency were studied both for laminar and turbulent flow regimes, and for micrometer and submicrometer particles. Optimized structures have been proven to perform better for fluid flow but they have a similar performance for particle transport.
\end{abstract}

\section{Introduction}

Flow of suspensions (particles) in complex flow structures occurs both in animate and inanimate systems [1], and is of interest in many fields of science and technology [2]. Examples include aerosol sampling devices [3], particle-, fibre- and membrane-based filtration technologies [2,4,5], but also micro-mixers technology for aerosol processing [6]. T-shaped mixers are widely used because of their simple geometry and advantages in comparison to batch-operated mixer [7]. Methodologies used in engineering frequently involve the use the aerosol flows, which have to be quick mixed or rapid delivered to a target $[7,8]$.

The aim of this work is to numerically study the fluid flow and aerosol transport in T-shaped structures. Hess [9] and Murray [10] suggested that an optimum relationship exists between consecutive diameters of bifurcating tubes that minimizes the power to maintain the laminar flow (allometric scaling law). Bejan's constructal theory [11], based on resistance minimization, provided a theoretical basis for this allometric law and extended it to consecutive tube lengths of bifurcating tubes under laminar and turbulent flows [12,13].

A comprehensive review of allometric laws in bifurcating flow structure is provided by $[14,15]$. Therefore, a systematic study was performed to compare the performance of geometry optimized structures and not optimized structures in the regard of flow resistance and also penetration efficiency of submicrometer and micrometer particles. 


\section{Model And Numerical Solution}

T-Shaped Flow Structure. We simulate fluid and particles suspension flows through 4 t-shaped structures with circular and square cross-section (see Fig. 1).
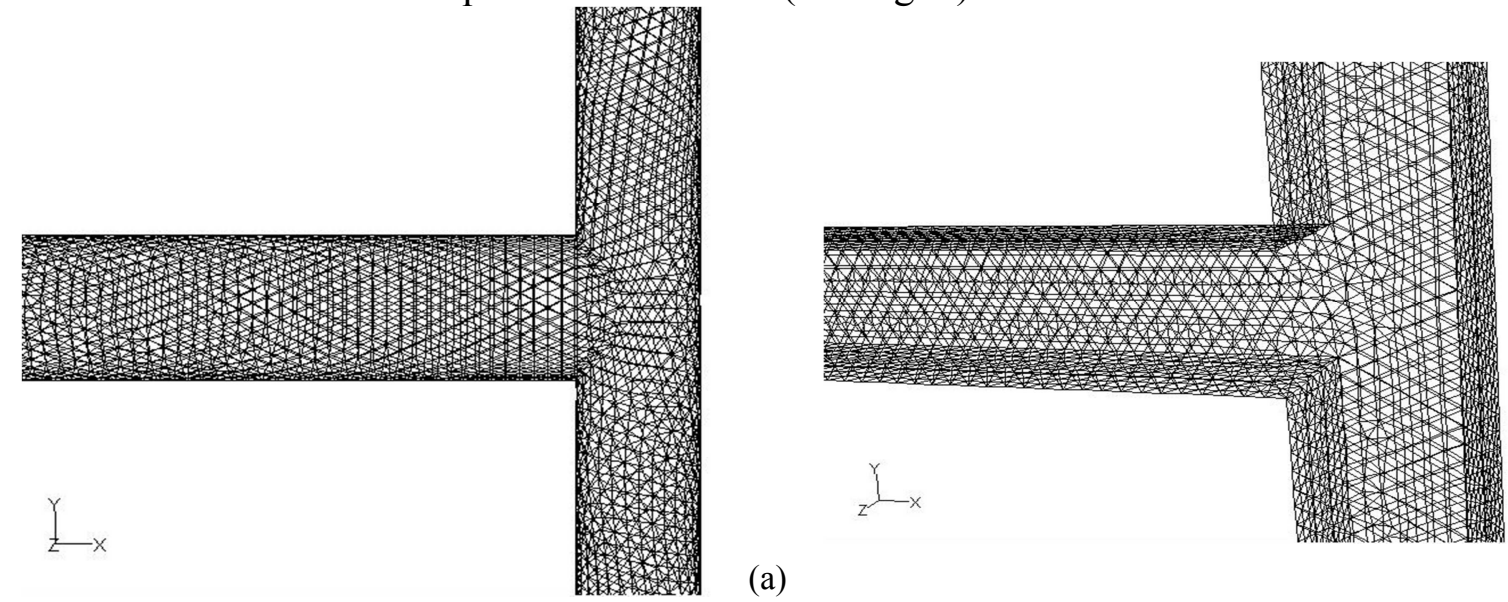

(a)

(b)

Figure 1. Symmetric T-shaped structures with circular (a) and square (b) cross-section.

The flow resistance in bifurcated pipes can be minimized under some special pipe design. Consider that the area covered by the geometry and the total volume allocated to the piping system are kept constant [11-16]. For laminar flow, the minimum flow resistance is achieved if the diameters and the lengths of consecutive pipes in a bifurcation are related as [14]

$d_{h 1}^{3}=0.5\left[\left(1+\lambda_{d}^{3}\right) d_{h 2}^{3}+\left(1+\lambda_{d}^{-3}\right) d_{h 3}^{3}\right]$

$L_{1}^{3}=0.5\left[\left(1+\lambda_{L}^{3}\right) L_{2}^{3}+\left(1+\lambda_{L}^{-3}\right) L_{3}^{3}\right]$

with $\lambda_{d}=d_{h 3} / d_{h 2}$ and $\lambda_{L}=L_{3} / L_{2}$. For symmetric pipes $\left(\lambda_{d}=\lambda_{L}=1\right)$, these equations become

$\frac{d_{h 1}}{d_{h 2}}=2^{1 / 3}$

$\frac{L_{1}}{L_{2}}=2^{1 / 3}$

Equation (3) is nothing more than the Hess-Murray law. For turbulent flow, the diameters and lengths of consecutive symmetric pipes were also optimized by Bejan et al. [12], and the following expressions were obtained

$$
\begin{aligned}
& \frac{d_{h 1}}{d_{h 2}}=2^{3 / 7} \\
& \frac{L_{1}}{L_{2}}=2^{1 / 7}
\end{aligned}
$$

Notice also that the geometric ratio of $d_{h} / L$ is preserved in going from each pipe to its branch in laminar flow (i.e., each pipe is geometrically similar to its tributary or collector). For turbulent flow, the constructal law shows that the geometric ratio of $d_{h} / L^{3}$ is preserved in going from each duct to its branch (i.e. $d_{h 1} / L_{1}{ }^{3}=d_{h 2} / L_{2}{ }^{3}$ ). 
Significance of junction losses on the geometry of bifurcation is obtained based on the svelteness number, $S v$, which is given by the ratio between the external length scale and the internal length scale of the system $[14,16]$.

$$
S v=\frac{\sum_{i} L_{i}}{\left(\sum_{i} L_{i} d_{h i}\right)^{1 / 2}}
$$

Table 1. Geometric characteristics of symmetric T-shaped structures (R: circular cross-section; S: square cross-section; C: constructal (i.e., designed according to Eqs. (3)-(6))

\begin{tabular}{|c|c|c|c|c|c|c|c|c|}
\hline Regime & Pipe geometry & Name & D1 (cm) & D2-D3 (cm) & L1 (cm) & L2-L3 (cm) & Area $\left(\mathrm{cm}^{2}\right)$ & Volume $\left(\mathrm{cm}^{3}\right)$ \\
\hline \multirow{4}{*}{ Laminar } & \multirow{2}{*}{ Round } & $\mathrm{R}$ & 0.44 & 0.44 & 5.35 & 5.35 & \multirow{4}{*}{57.2} & \multirow{4}{*}{2.4} \\
\hline & & $\mathrm{RC}$ & 0.50 & 0.40 & 6.00 & 4.76 & & \\
\hline & \multirow{2}{*}{ Square } & $\mathrm{S}$ & 0.44 & 0.44 & 5.35 & 5.35 & & \\
\hline & & $\mathrm{SC}$ & 0.45 & 0.36 & 6.00 & 4.76 & & \\
\hline \multirow{4}{*}{ Turbulent } & \multirow{2}{*}{ Round } & $\mathrm{R}$ & 0.44 & 0.44 & 5.35 & 5.35 & \multirow{4}{*}{57.2} & \multirow{4}{*}{2.4} \\
\hline & & $\mathrm{RC}$ & 0.52 & 0.39 & 5.62 & 5.09 & & \\
\hline & \multirow{2}{*}{ Square } & $\mathrm{S}$ & 0.39 & 0.39 & 5.35 & 5.35 & & \\
\hline & & $\mathrm{SC}$ & 0.46 & 0.34 & 5.62 & 5.09 & & \\
\hline
\end{tabular}

Table 1 displays the geometric characteristics of each structure. Data depicted in this table show that the svelteness number is about 6 for all the geometries. Therefore, the junction losses have minor sizable effects on the diameter ratio because $S v$ is lower than the square root of 10 [17].

Numerical Procedure for Fluid Flow and Particle Dynamics. The steady state flow field is defined by the 3D Navier-Stokes equations together with the standard k- $\varepsilon$ model of turbulence and solved numerically using the code FLUENT [18] which is based on a finite volume method. The airflow is assumed to be incompressible. The non-slip boundary conditions are set along the walls. The flat velocity profile is prescribed at the inlet while pressure outlet boundary conditions are set at the outlet.

The geometries and correspondent grids were generated in Gambit [18]. The accuracy of our numerical simulations was validated with respect to refinement and spatial resolution of the grid based on the methodology proposed by Roache [19] and Sidi [20]. Grids with 9740-9810 cells and 5257-5268 nodes are found to be appropriate for the present study.

Airflows for Reynolds numbers, based on the hydraulic diameter of the inlet tube, ranging from 20 to 5000, were considered. Once the solution of air flow was obtained, each particle trajectory was then calculated. All particles are assumed to be spherical. The trajectory of each particle is predicted by integrating the force balance on the particle $[4,8,21]$

$$
\frac{d u_{p}}{d t}=\frac{1}{\rho_{p}}\left[\frac{18 \mu}{d_{p}^{2}} \frac{c_{d} \operatorname{Re}}{24}\left(u-u_{p}\right)+\frac{1}{2} \rho \frac{d}{d t}\left(u-u_{p}\right)+\rho u_{p} \frac{d u}{d t}+g\left(\rho_{p}-\rho\right)+F_{B r}\right]
$$

Here the first right-hand term represents the drag force, the second right-hand term is the "virtual mass" force required to accelerate the fluid surrounding the particle, the third right-hand term is the force due to pressure gradient in the fluid, the fourth right-hand term is the gravitational force, the fifth right-hand term denotes a force arising from Brownian collisions [2], $u$ is the fluid velocity, $u_{p}$ is particle velocity, $R e$ is the Reynolds number, $\rho_{p}$ is the particle density $c_{d}$ is drag coefficient, $\rho$ is the fluid density, $\mu$ is the dynamic viscosity, $t$ is the time and $g$ is the gravity constant. The components of random force (third fifth-hand term) are evaluated at each time step. In this setup, particles that touch the solid walls stick to it at first collision. 
Particles (density $1 \mathrm{~kg} / \mathrm{m}^{3}$, diameters between 0.1 and $100 \mu \mathrm{m}$ ) are introduced at the inlet of the Tshaped structure, and tracked through the geometry until they are trapped on the solid walls or escape through the outlet of the structure.

\section{Results}

The velocity and pressure fields in the T-shaped structures $\mathrm{R}, \mathrm{RC}, \mathrm{S}$ and $\mathrm{SC}$ were numerically obtained for a range of Reynolds number $\left(R e=\rho u d_{h} / \mu\right)$ between 20 and 5000. The resistance through each structure can be modeled in terms of Darcy-Weisbach friction factor [2]

$$
\Delta p=\frac{f}{2} \frac{L}{d_{h}} \rho u^{2}
$$

Here $u$ is the inlet velocity, $p$ is the pressure, $d_{h}$ is the hydraulic diameter and $f$ is the DarcyWeisbach friction factor. Equation (9) is successfully correlated with the data of our numerical simulations and the friction factor $\mathrm{f}$ is obtained from the best fit of these data. The results are presented in Table 2. The table shows that the friction factor for constructal T-shaped structures (RC, SC) is smaller than for configurations $\mathrm{R}$ and S. Besides, structures with a circular crosssection present lower friction factor than structures with square cross-section.

Table 2. Darcy-Weisbach friction factor for the T-shaped structures.

\begin{tabular}{cccc}
\hline Geometry & Regime & Sv & $\begin{array}{c}\text { Friction } \\
\text { Factor }\end{array}$ \\
\hline R & & 6.1 & 0.76 \\
RC & Laminar & 6.0 & 0.52 \\
S & & 6.1 & 0.88 \\
SC & & 6.3 & 0.61 \\
R & & 6.0 & 10.46 \\
RC & Turbulent & 6.0 & 5.65 \\
S & & 6.4 & 12.47 \\
SC & & 6.4 & 6.83 \\
\hline
\end{tabular}

Figure 2 shows the air velocity within RC and R structure under laminar and turbulent flow. In spite of the area covered by the geometry, the total volume allocated to the piping system and svelteness number are similar, profile of velocities (and pressure) is different. The major differences are in the bifurcation. For $R e=20$, the constructal configuration (RC) presents more uniform velocity. For $R e=3500$, the plot reveals a well-defined "preferential channel" having also a more uniform velocity.

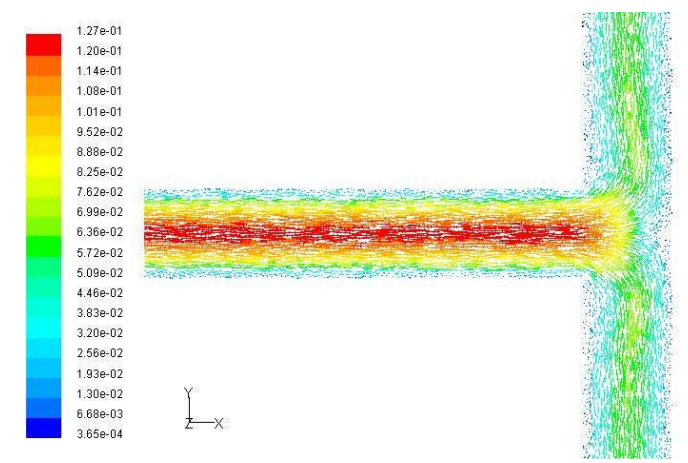

(R)

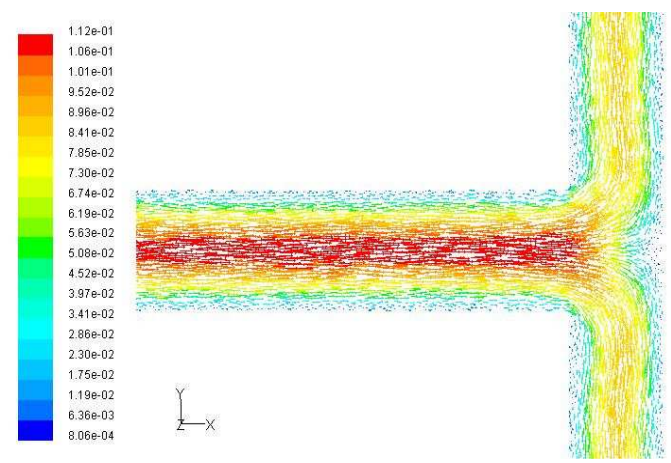

Figure 2a. Velocity magnitude (middle iso-surface) for R and RC structure (Reynolds 20) 


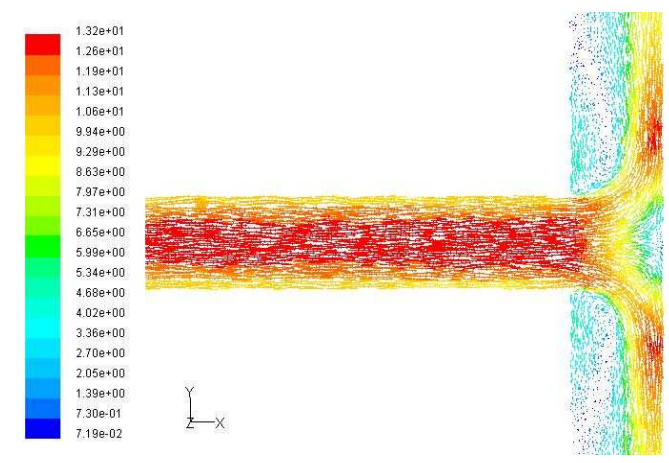

(R)

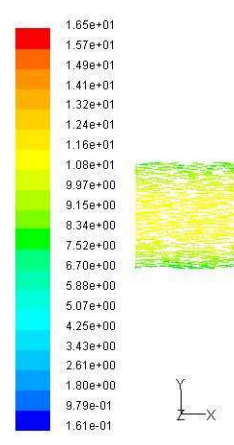

$(\mathrm{RC})$

Figure 2B. Velocity magnitude (middle iso-surface) for R and RC structure (Reynolds 3500)

The penetration efficiency, $\eta$, defined as the number of particles counted at the outlet sections of the T-shaped structure divided by the number of particles at the inlet, is depicted in Table 3 . It is observed that the penetration efficiency is mainly determined by the diameter of the particles. Constructal and non-constructal T-shaped structures present similar values of penetration efficiency but structures with circular cross-section pipes show higher values of $\eta$ than square cross-section pipes. The table also documents that under laminar and turbulent flow regime, $\eta$ is almost insensitive to the Reynolds number. For turbulent flow all particles with diameters of 10, 50 and $100 \mu \mathrm{m}$ deposit inside the T-shaped structure. The deposition of these particles is mainly induced by inertial particle deposition where the structure has sharp angles: particles cannot follow the streamlines due to their inertia, and impact on the surface of the wall where they adhere.

Table 3. The penetration efficiency versus the particle diameter for $\mathrm{R}, \mathrm{RC}, \mathrm{S}$ and $\mathrm{SC}$ structures.

\begin{tabular}{|c|c|c|c|c|c|c|c|c|c|c|c|c|c|c|c|c|}
\hline \multirow[t]{2}{*}{$\mathbf{R e}$} & \multicolumn{4}{|c|}{$\begin{array}{c}R(S v=6.1) \\
d_{p}(\mu \mathrm{m})\end{array}$} & \multicolumn{4}{|c|}{$\begin{array}{c}\mathrm{RC}(\mathrm{Sv}=6.0) \\
\mathrm{d}_{\mathrm{p}}(\mu \mathrm{m})\end{array}$} & \multicolumn{4}{|c|}{$\begin{array}{c}S(S v=6.1) \\
d_{p}(\mu m)\end{array}$} & \multicolumn{4}{|c|}{$\begin{array}{c}S C(S v=6.3) \\
d_{p}(\mu \mathrm{m})\end{array}$} \\
\hline & 0.1 & 10 & 50 & 100 & 0.1 & 10 & 50 & 100 & 0.1 & 10 & 50 & 100 & 0.1 & 10 & 50 & 100 \\
\hline 20 & 0.94 & 0.96 & 0.47 & 0.00 & 0.90 & 0.93 & 0.45 & 0.00 & 0.87 & 0.88 & 0.27 & 0.02 & 0.89 & 0.88 & 0.35 & 0.01 \\
\hline 40 & 0.90 & 0.91 & 0.05 & 0.00 & 0.91 & 0.93 & 0.10 & 0.00 & 0.87 & 0.83 & 0.05 & 0.00 & 0.90 & 0.87 & 0.09 & 0.01 \\
\hline 60 & 0.90 & 0.92 & 0.01 & 0.00 & 0.90 & 0.92 & 0.01 & 0.00 & 0.88 & 0.83 & 0.03 & 0.00 & 0.89 & 0.86 & 0.03 & 0.00 \\
\hline 80 & 0.90 & 0.88 & 0.00 & 0.00 & 0.91 & 0.90 & 0.00 & 0.00 & 0.91 & 0.84 & 0.02 & 0.00 & 0.89 & 0.88 & 0.02 & 0.00 \\
\hline \multirow[t]{2}{*}{$\operatorname{Re}$} & \multicolumn{4}{|c|}{$\begin{array}{c}R(S v=6.0) \\
d_{p}(\mu m)\end{array}$} & \multicolumn{4}{|c|}{$\begin{array}{c}\mathrm{RC}(\mathrm{Sv}=6.0) \\
\mathrm{d}_{\mathrm{p}}(\mu \mathrm{m})\end{array}$} & \multicolumn{4}{|c|}{$\begin{array}{c}S(\mathrm{~Sv}=6.4) \\
d_{p}(\mu \mathrm{m})\end{array}$} & \multicolumn{4}{|c|}{$\begin{array}{c}S C(S v=6.4) \\
d_{p}(\mu \mathrm{m})\end{array}$} \\
\hline & 0.1 & 10 & 50 & 100 & 0.1 & 10 & 50 & 100 & 0.1 & 10 & 50 & 100 & 0.1 & 10 & $\mathbf{5 0}$ & 100 \\
\hline 1500 & 0.91 & 0.00 & 0.00 & 0.00 & 0.94 & 0.00 & 0.00 & 0.00 & 0.81 & 0.04 & 0.00 & 0.00 & 0.85 & 0.00 & 0.00 & 0.00 \\
\hline 2500 & 0.92 & 0.00 & 0.00 & 0.00 & 0.93 & 0.00 & 0.00 & 0.00 & 0.84 & 0.01 & 0.00 & 0.00 & 0.88 & 0.00 & 0.00 & 0.00 \\
\hline 3500 & 0.92 & 0.00 & 0.00 & 0.00 & 0.93 & 0.00 & 0.00 & 0.00 & 0.87 & 0.00 & 0.00 & 0.00 & 0.91 & 0.00 & 0.00 & 0.00 \\
\hline 5000 & 0.92 & 0.00 & 0.00 & 0.00 & 0.92 & 0.00 & 0.00 & 0.00 & 0.84 & 0.00 & 0.00 & 0.00 & 0.90 & 0.00 & 0.00 & 0.00 \\
\hline
\end{tabular}

Deposition of particles may be analyzed in terms of the Peclet number, $P e$. This dimensionless number measures the relative importance of convection to diffusion and is given by [1,2]

$P e=\frac{u d_{h}}{D}$

and the particle diffusion coefficient $D$ is calculated from

$D=\frac{K T c_{c}}{3 \pi \mu d_{p}}$

where $K$ is the Boltzmann constant, $T$ is the absolute fluid temperature and the $c_{c}$ is the Cunningham nonslip correction. As the Peclet number increases, the diffusion process becomes less important. For high $P e$ diffusion losses are very small and deposition is inside the inertia-dominated regime. 
Figure 3 shows the penetration efficiency versus the Peclet number. The penetration efficiency depicted in this figure reveal a small decrease up to $P e \sim 10^{9}$. After, the $\eta$ versus $P e$ curve starts to decrease abruptly which means that the inertia-dominated deposition regime starts to be prevalent. The particle dynamics at low Peclet numbers should be characterized by higher residence times within the flow structure [2]. This is an important factor in several industrial applications. Figure 4 shows clearly a decrease of the residence time with the Peclet number. An abrupt decrease of the penetration efficiency (i.e., the start of inertia-dominated deposition regime) is linked to a very small residences time inside the T-shaped structure.

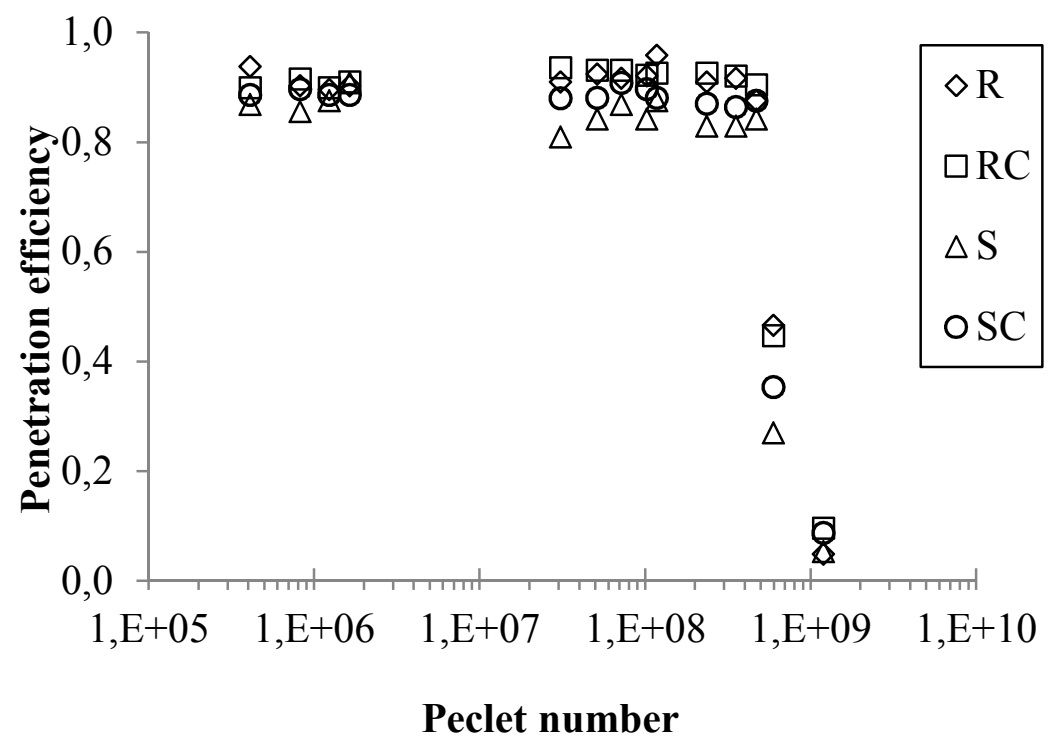

Figure 3. The penetration efficiency versus the Peclet number.

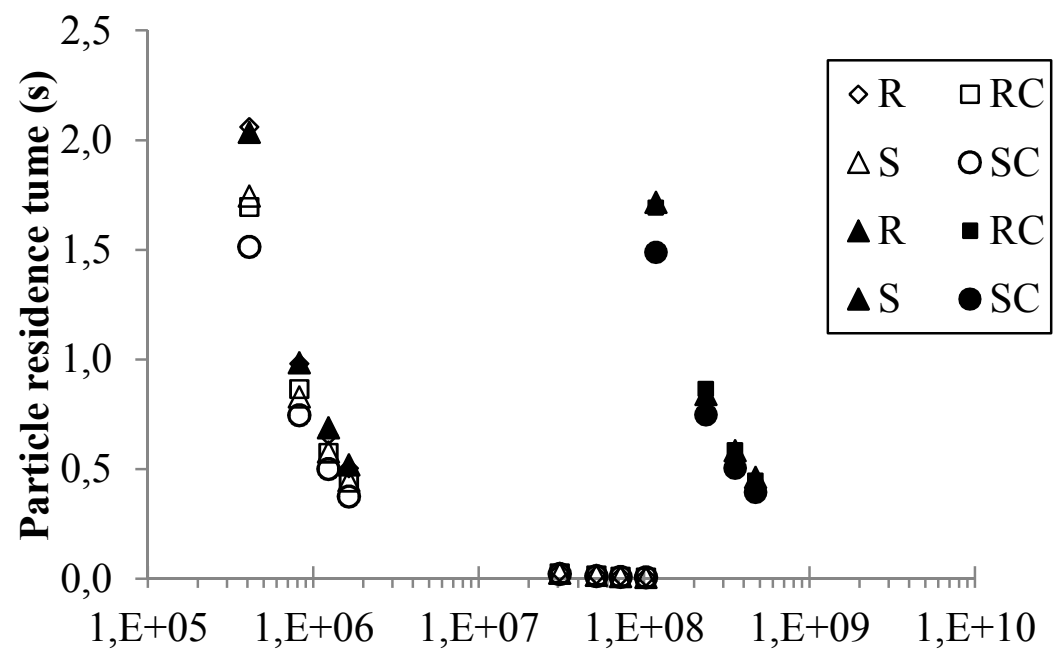

Peclet number

Figure 4. Average particle residence times versus the Peclet number (white-filled symbols $0.1 \mu \mathrm{m}$, black-filled symbols $10 \mu \mathrm{m})$ 


\section{Conclusions}

This study shows that constructal-shaped structures are the easiest path (least resistance) that connects one point with an infinity of points (line, area, or volume) and vice-versa. Beside, Tshaped structures with circular cross-section have less resistance to flow than square cross-section. Constructal and non-constructal T-shaped structures present similar values of penetration efficiency but structures with circular cross-section show higher values than square cross-section pipes. The penetration of particles is almost insensitive to the Reynolds number but is strong dependent on the Peclet number. The residence time within the T-shaped structure is related with the Peclet number is also provided.

\section{References}

[1]. A. F. Miguel: "Lungs as a Living Porous Medium: flow structure and aerosol transport", in Heat and Mass Transfer in Porous Media, edited by J.M.P.Q Delgado, New York: Springer, 2012, pp.115-138

[2]. A. Bejan, I. Dincer, L. Lorente, A. F. Miguel, and A. H. Reis: Porous and Complex Flow Structures in Modern Technologies, New York: Springer, 2004

[3]. S.K. Friedlander, Smoke, Dust and Haze, New York: Wiley, 1977

[4]. A. Serrenho and A. F. Miguel: Defect and Diffusion Forum 315, 871-876, (2011)

[5]. A. F. Miguel and A. H. Reis: Journal of Porous Media 9, 731-744 (2006)

[6]. N. Kockmann, M. Engler and P. Woias: "Convective mixing and chemical reactions in Tshaped micro reactors", in Proc. AIChE Annual Meeting, Austin, 2004, paper 330

[7]. N. Kockmann, S. Dreher, M. Engler and P. Woias: Microfluid Nanofluid 3, 581-589 (2007)

[8]. A. Serrenho and A. F. Miguel: Journal of Porous Media 12, 1129-1138 (2009).

[9]. W.R. Hess, "Das Prinzip des kleinsten Kraftverbrauches im Dienste hämodynamischer Forschung Archiv für Anatomie und Physiologie”, Physiologische Abteilung, 1914

[10]. C. D. Murray, Proc. Natl. Acad. Sci. USA 12, 207-214, (1926)

[11]. A. Bejan: "Shape and structure from engineering to nature" Cambridge: Cambridge University Press 2000.

[12]. A. Bejan, L.A.O. Rocha and S. Lorente: Int. J. Therm. Sci. 39, 949-960, 2000

[13]. A. F. Miguel: International Journal of Design \& Nature and Ecodynamics 5, 2010, 230-241

[14]. A. F. Miguel: "Tree-shaped Flow Structures Viewed from the Constructal Theory Perspective", in Single and two-Phase Flows on Chemical and Biomedical Engineering, edited by R. Dias et al., Bentham Science Publishers, 2012, pp. 195-213

[14]. A. F. Miguel: J. Fluids Structures 26, 2010, 330-335

[16]. A. F. Miguel: Defect and Diffusion Forum 297-301, 2010, 408-412

[17]. W. Wechsatol, S. Lorente and A. Bejan: Int. J. Heat Mass Transfer 49, 2006 2957-2964

[18]. FLUENT 6 User's Guide, Fluent Inc., 2003

[19]. P. J. Roache: Verification and Validation in Computational Science and Engineering, Albuquerque: Hermosa Publishers, 1998

[20]. A. Sidi: Practical Extrapolation Methods: Theory and Applications, Cambridge: Cambridge Monographs on Applied and Computational Mathematics, 2002

[21]. A. F. Miguel and A. M. Silva: Journal of Aerosol Science 34, 2003, S667-S668 